\title{
One-Week Nebulization of Mycobacterium vaccae Can Protect Against Pulmonary Respiratory Syncytial Virus Infection in Balb/c Mice
}

\author{
Xiao-Hong Jiang, PhD, ${ }^{1, \star}$ Chao-Qian Li, PhD, ${ }^{1, *}$ Guang-Yi Feng, MD, ${ }^{2}$ \\ Ming-Jie Luo, MD, ${ }^{3}$ and Qi-Xiang Sun, $\mathrm{MD}^{4}$
}

\begin{abstract}
Background: Respiratory syncytial virus (RSV) infection is the most common cause of acute lower respiratory tract infection in children, leading to their death. Currently, no effective prevention and treatment methods for RSV infection are available. RSV and many other unknown viruses pose a serious threat to human health. Our previous study demonstrated that Mycobacterium vaccae nebulization can protect against allergic asthma. As RSV infection and asthma are closely related, we hypothesized that $M$. vaccae could protect against pulmonary RSV infection. Therefore, we evaluated the effect of $M$. vaccae on RSV infection in Balb/c mice.

Methods: The mice were randomized into three groups: normal, RSV, and M. vaccae. One week before the RSV infection model was established, the mice in the $M$. vaccae group were nebulized with $M$. vaccae. On the fourth day after RSV infection, airway responsiveness, airway inflammation, pulmonary RSV infection, mRNA levels of pulmonary toll-like receptor (TLR) 7 and TLR8, and pulmonary NF09, acetylcholine, and epidermal growth factor regulator (EGFR) expression levels in all mice were measured.

Results: The airway inflammation in the M. vaccae group was alleviated compared with that in the RSV group. In the $M$. vaccae group, the pulmonary mRNA level of RSV and the pulmonary expression levels of NF09, acetylcholine, and EGFR were decreased considerably, whereas the mRNA levels of TLR7 and TLR8 were increased significantly.

Conclusions: One-week nebulization of $M$. vaccae can protect against RSV infection in Balb/c mice. The mechanism involves the regulation of neurotransmitters and expression of TLR7, TLR8, and EGFR.
\end{abstract}

Keywords: Mycobacterium vaccae, nebulization, neurotransmitters, RSV

\section{Introduction}

$\mathbf{R}$ ESPIRATORY VIRUS INFECTIONS are acute infectious diseases in humans, and their prevention and treatment remain challenging. ${ }^{(1)}$ Respiratory syncytial virus (RSV) is the most common pathogen infecting the lower respiratory tract of infants and adults worldwide. To date, only two medications for RSV prevention and treatment have been approved by the Food and Drugs Administration (FDA) of the United States. One of these drugs is palivizumab, ${ }^{(2)}$ the other is Ribavirin, but because of their high cost and underlying adverse effects, they are not available in many coun- tries. Research studies have demonstrated that severe RSV infections in infants are closely associated with asthma development in adults. ${ }^{(3-5)}$

Studies have confirmed that the Bacillus Calmette-Guérin (BCG) vaccine can protect against allergic bronchial asthma. ${ }^{(6)}$ Previously, we have demonstrated that Mycobacterium vaccae vaccine reduces airway hyper-responsiveness and protects against bronchial asthma in mice. ${ }^{(7)}$ The $M$. vaccae vaccine is mainly composed of mycobacterial proteins.

In addition to the nervous system, neurotrophins are expressed in peripheral tissues, including the lung tissue. ${ }^{(8)}$ Recent studies have found that neurogenic airway

\footnotetext{
${ }^{1}$ Department of Geriatric Respiratory Medicine, The First Affiliated Hospital of Guangxi Medical University, Nanning, China.

${ }^{2}$ Department of Respiratory Medicine, The Sixth Affiliated Hospital of Guangxi Medical University, Yulin, China.

${ }^{3}$ Department of Respiratory Medicine, Nanxishan Hospital of Guangxi Zhuang Autonomous Region, Guilin, China.

${ }^{4}$ The Graduate School of Guangxi Medical University, Nanning, China.

*These authors contributed equally to this work.
} 
inflammation plays an important role in the inflammatory mechanism of asthma. Nerve growth factor (NGF), brainderived neurotrophic factor (BDNF), neurofilaments (NFs), and acetylcholine are connected with this inflammation. Airway neurotrophic factors, such as BDNF, and their receptors were overexpressed in children with RSV infection. ${ }^{(9)}$ The quantity and distribution density of NFs indirectly reflect the density of nerve synapses, and thus reflect neural network conditions. Earlier studies have found that transplacental transmission of RSV from mother to offspring can lead to an increase in acetylcholine innervation in fetuses of rats.

Considering that RSV infection and asthma are closely related and that short-time nebulization of inactivated Mycobacterium phlei can prevent and treat asthma by upregulating interferon (IFN) levels and correcting Th1/Th2 imbalance, we sought to investigate whether such nebulization can protect from RSV and other respiratory virus infections. Thus, we established an RSV infection mouse model and investigated whether $M$. vaccae nebulization exerts preventive effects on RSV infection. In addition, we investigated the mechanisms of prevention at the level of neurotransmitters and the regulation of toll-like receptor (TLR)7, TLR8, and epidermal growth factor regulator (EGFR).

\section{Materials and Methods}

This study was performed in accordance with the Guide for the Care and Use of Laboratory Animals of the National Institutes of Health (NIH), and was approved by the Guangxi Medical University Animal Care and Use Committee (Protocol No. 20131002). All surgeries were performed under Nembutal anesthesia, and every effort was made to minimize the suffering of the experimental animals.

Mice

Eighteen pathogen-free female Balb/c mice (20-25 g) were purchased from the Medical Animal Center of Guangdong Province (Guangdong, China). They were maintained in an air-conditioned room (temperature: $23^{\circ} \mathrm{C} \pm 3^{\circ} \mathrm{C}$, humidity: $55.5 \% \pm 1 \%)$.

\section{Experimental protocol}

Mice were randomized into three groups: normal, RSV, and $M$. vaccae. One week before the RSV infection model was established, the $M$. vaccae group was nebulized with $22.5 \mu \mathrm{g}$ M. vaccae (Anhui Longkema Biological Pharmaceutical Co., Anhui, China) mixed with $10 \mathrm{~mL}$ phosphatebuffered saline (PBS) once daily for five consecutive days. The normal and RSV groups were nebulized with PBS. The RSV and $M$. vaccae groups were infected nasally with $50 \mu \mathrm{L}$ of $2 \times 10^{6}$ plaque forming units (PFUs) RSV once daily for three consecutive days, whereas the normal group was received with PBS. Lung tissue samples were harvested from all mice on the fourth day after RSV infection (Fig. 1).

\section{$R S V$ infection}

The RSV long strain was obtained from the American Type Culture Collection (ATCC, Manassas, VA). The virus was amplified and purified. The mice were anesthetized subcutaneously with chloral hydrate $(0.35 \mathrm{mg} / \mathrm{g}$ body weight $)$ and infected with $50 \mu \mathrm{L}$ of $2 \times 10^{6}$ PFU RSV long strain diluted in PBS. This was performed through intranasal inoculation once a day for three consecutive days.

\section{Airway responsiveness measurement}

Airway responsiveness was measured using Buxco FinePointe Non-Invasive Airway Machine (St. Paul, MN) on the fourth day after RSV infection. After calibration and 5 minutes of adaptation, the mice were nebulized with $20 \mu \mathrm{L}$ PBS and methacholine (Sigma) at concentrations of 6.25 , 12.5 , and $25 \mathrm{mg} / \mathrm{mL}$ for 30 seconds each. Data were recorded for 3 minutes, and the mice recovered after 4 minutes. The results were analyzed automatically at the end of the experiment. The airway responsiveness is presented as sRaw (specific airway resistance).

\section{Specimen collection}

The mice were anesthetized using an intraperitoneal injection of $1 \%$ nembutal $(50 \mathrm{mg} / \mathrm{kg}$ weight $)$, bled through an orbital puncture, and fixed on their backs. The lungs were lavaged using $500 \mu \mathrm{L}$ ice-cold PBS three times, and the

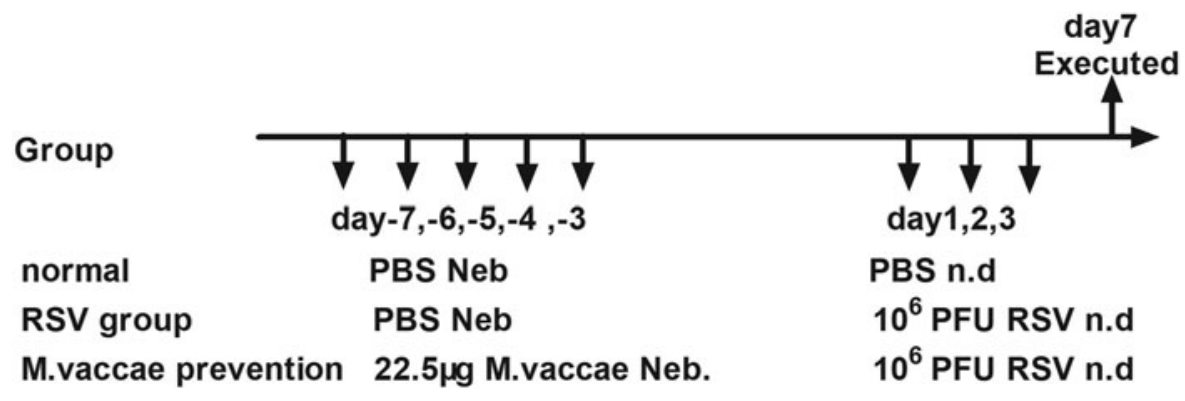

FIG. 1. Experimental protocol. Mice were randomized into three groups: normal, RSV, and Mycobacterium vaccae prevention (M. vaccae group) groups. One week before the RSV infection model was established, the $M$. vaccae group was nebulized with $22.5 \mu \mathrm{g} M$. vaccae mixed with $10 \mathrm{~mL}$ PBS once daily for five consecutive days. The normal and RSV groups were nebulized with PBS. The RSV and $M$. vaccae groups were nasal infected with $50 \mu \mathrm{L} 2 \times 10^{6}$ PFU RSV once daily for three consecutive days, the normal group with PBS. All mice were harvested on the fourth day after RSV infection. PFU, plaque forming unit; PBS, phosphate-buffered saline; $\mathrm{RSV}$, respiratory syncytial virus. 
bronchoalveolar lavage fluid (BALF) was collected in microcentrifuge tubes on ice. The recovery of BALF was $>80 \%$. The BALF was centrifuged to detect cytokines in the supernatant. The right upper lobes were fixed in formalin for histology, and the left lobes were cut into pieces and digested with $0.1 \%$ collagenase IV (Sigma) for 45 minutes to produce a single-cell suspension. Lymphocytes from the single-cell suspension were separated by flow cytometry using mouse tissue lymphocytes separation liquid (Haoyang, Tianjing, China).

\section{Histology}

The lung tissue was fixed in $10 \%$ formaldehyde solution for 12 hours. After gradient alcohol dehydration, dewaxing with xylene, and paraffin embedding, the tissue was sectioned at a thickness of $3 \mu \mathrm{m}$. Both hematoxylin-eosin (HE) staining and periodic-acid Schiff staining were performed to observe the alveolar structure, infiltration of inflammatory cells around the airway, coloration of the airway epithelium, distribution of goblet cells, and secretion of mucus.

\section{Immunohistochemistry assay for NF09, acetyl choline, and EGFR in the lung tissue}

Immunohistochemistry was used to detect NF09, acetyl choline, and EGFR proteins, as described below: (1) preparation of lung tissue sections: the lung tissue was fixed for 12-24 hours in 10\% formaldehyde solution, treated with gradient alcohol dehydration, dewaxed with xylene, and embedded in paraffin. A $3 \mu \mathrm{m}$-thick transverse section was obtained. (2) Immunohistochemical detection: the lung tissue slice was baked in a thermostat at $60^{\circ} \mathrm{C}$ for 3 hours, then dewaxed with xylene, and washed with alcohol and water. After dewaxing, the lung tissue was repaired with citric acid solution at a high temperature and pressure for 2 minutes, incubated for 15 minutes in $3 \% \mathrm{H}_{2} \mathrm{O}_{2}$ for blocking the endogenous peroxidase, closed for 10 minutes with goat serum to reduce nonspecific staining, and incubated overnight at an appropriate concentration of the primary antibody at $4^{\circ} \mathrm{C}$. The next morning, the slice was reheated in a $37^{\circ} \mathrm{C}$ constant temperature box for 30 minutes and soaked completely with PBS. Then, the secondary antibody (Abcam, Cambridge, United Kingdom) was added after removing the PBS solution. Next, it was incubated with horseradish peroxidase at room temperature for 15 minutes and rinsed with PBS. After incubating at room temperature for 10 minutes, freshly prepared diaminobenzidine (DAB) (20:1:1) was applied for coloring. Every sample was washed with PBS to terminate coloring, and finally, the samples were counterstained with hematoxylin for 40 seconds. Next, the sections were dehydrated with $80 \%, 90 \%$, and $100 \%$ alcohol, and the sections were mounted using neutral gum. The slides were then clustered and photographed with a microscope. Each slice was photographed under the microscope for 400 times nonrepetitively. The images were analyzed using the ImagePro Plus 6 software. The average integral light density [integral optical density (IOD) mean] was measured.

\section{Measuring mRNA expression of RSV, TLR7, and TLR8}

The mRNA expression levels of RSV, TLR7, and TLR8 were measured using polymerase chain reaction (PCR). The
PCR primers were designed as follows: RSV upstream: GG TTTTTTGTTAGGTGTTGGATCTG, RSV downstream: GCCTTGTTTGTGGATAGTAGAGCA; TLR7 upstream: TTAGGAAACCATCGAAACCCAAAG, TLR7 downstream: CCTCTGGAACCAGTGCCATC; TLR8 upstream: GGTTGCATTCTGCAATCACAAG, TLR8 downstream: GGTTGCATTCTGCAATCACAAG; Gapdh upstream: TGT GTCCGTCGTGGATCTGA, Gapdh downstream: TTGCTG TTGAAGTCGCAGGAG (TaKaRa Biotechnology, Japan).

\section{Statistical analysis}

Statistical analyses were performed using GraphPad Prism version 8.00 for Windows. $T$-test was used to compare the data between groups.

\section{Results}

\section{Effects of M. vaccae nebulization on airway hyper-responsiveness}

The airway hyper-responsiveness of mice in the RSV and M. vaccae groups did not differ significantly (Fig. 2).

\section{Histology}

In both the RSV and $M$. vaccae groups, airway epithelial cells were swollen, plicae mucosae were increased, cilia were disordered, bronchial mucous membranes were broken, many inflammatory cells had infiltrated bronchioles, vessels had an alveolar space and interalveolar septum, the alveolar wall was thickened showing hyperemia and dropsy, the basilar membrane was thickened, and smooth muscle showed hyperplasia. These signs demonstrated that $M$. vaccae nebulization could not alleviate lung inflammation induced by RSV infection (Fig. 3).

\section{Effects of M. vaccae nebulization on lung RSV infection assessed by electron microscopy}

Lung RSV infection decreased considerably after $M$. vaccae nebulization (Fig. 4).

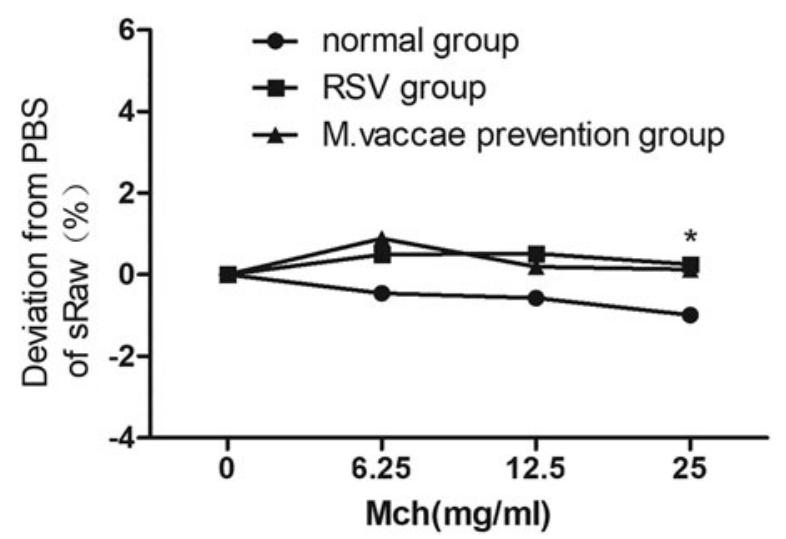

FIG. 2. Airway hyper-responsiveness results. ${ }^{*} p<0.05$, compared with normal group. 


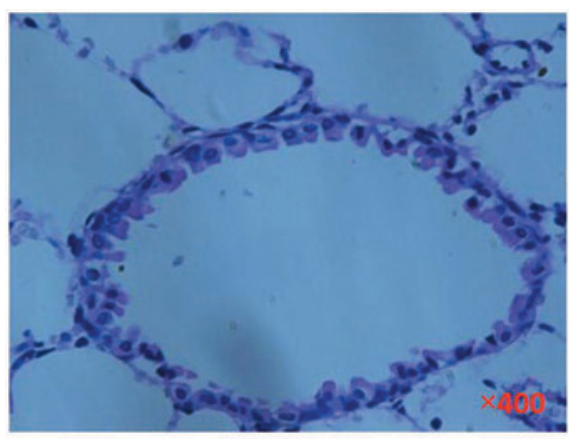

Normal group

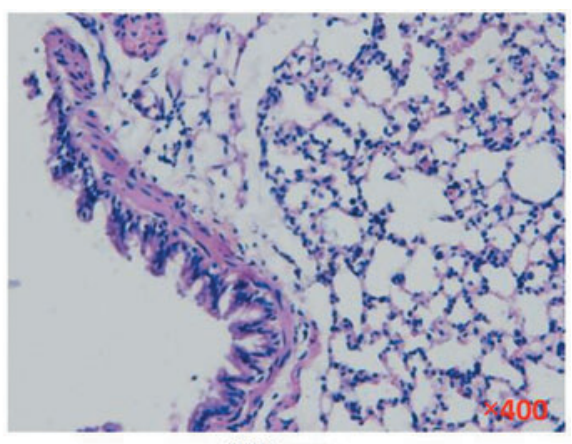

RSV group

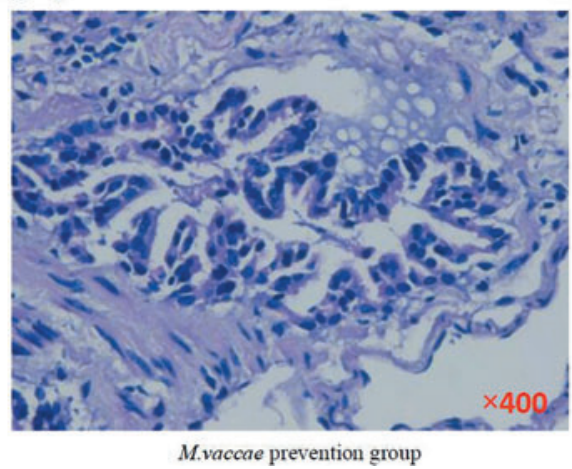

FIG. 3. Histology $(\times 400)$. Both in the RSV control and in Mycobacterium vaccae group, there was cellular swelling of airway epithelia, the plicae mucosae were increased, cilia were disordered, the bronchial mucous membranes were broken, many inflammatory cells infiltrated bronchioles, vessels had an alveolar space and interalveolar septum, the alveolar wall was thickened showing hyperemia and dropsy, the basilar membrane was thickened, smooth muscle showed hyperplasia. demonstrating that $M$. vaccae nebulization cannot alleviate lung inflammation induced by RSV infection. Color images are available online.

\section{Effects of M. vaccae nebulization on lung anti-RSV} expression detected by immunofluorescence

The expression of anti-RSV increased in the lungs of the RSV group, while it decreased in the M. vaccae group (Fig. 5).

\section{Effects of M. vaccae nebulization on RSV mRNA level detected by quantitative PCR}

Mice lung RSV mRNA level decreased considerably after $M$. vaccae nebulization, demonstrating that $M$. vaccae can effectively protect against RSV infection (Fig. 6).

Effects of M. vaccae nebulization on lung NF09, acetylcholine, and EGFR expression in mice lungs detected by immunohistochemistry

The expression of NF09, acetylcholine, and EGFR in the lungs increased significantly in the RSV group, whereas it decreased considerably in the $M$. vaccae group (Figs. 7-9).

\section{Effects of M. vaccae nebulization on the mRNA expression levels of TLR7 and TLR8 detected by quantitative PCR}

The mRNA levels of TLR7 and TLR8 increased significantly after $M$. vaccae nebulization (Fig. 10).

\section{Discussion}

Developing an effective preventive method against RSV that has few adverse effects and good compliance metrics is crucial. It will be more helpful if this method is effective against the majority of viruses, preferably all viruses. Currently, developing such a method is a major problem that needs to be tackled on an urgent basis. The two recommended medications for RSV prevention and treatment are palivizumab $^{2}$ and Ribavirin, Palivizumab, a human monoclonal antibody against the RSV F protein. It is used in clinical settings to distinguish between type A and type B. It is effective in reducing the frequency of hospitalizations due to RSV infection in children, and is approved as a preventive measure against severe RSV infection in infants. However, owing to its high cost, palivizumab is not available in many countries as a standard treatment. ${ }^{(10)}$ Recent studies have found that children who received palivizumab had no beneficial effects on long-term respiratory morbidity, airway reactivity, or allergic parameters. ${ }^{(11)}$ Ribavirin is another drug that is used to treat RSV in the clinic. However, owing to its limited benefits and underlying adverse effects, it is not recommended for routine use in children by the American Academy of Pediatrics. Another study indicated that motavizumab could not reduce RSV load and severity of disease. ${ }^{(12)}$

Recent studies have found that neurogenic airway inflammation plays an important role in the inflammatory 


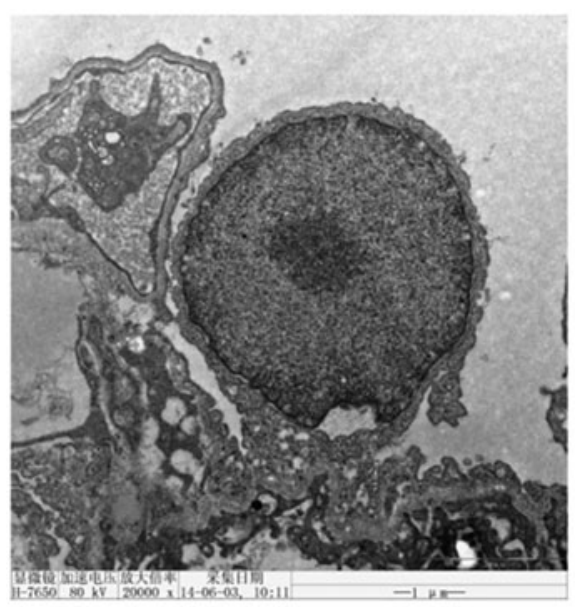

Normal group

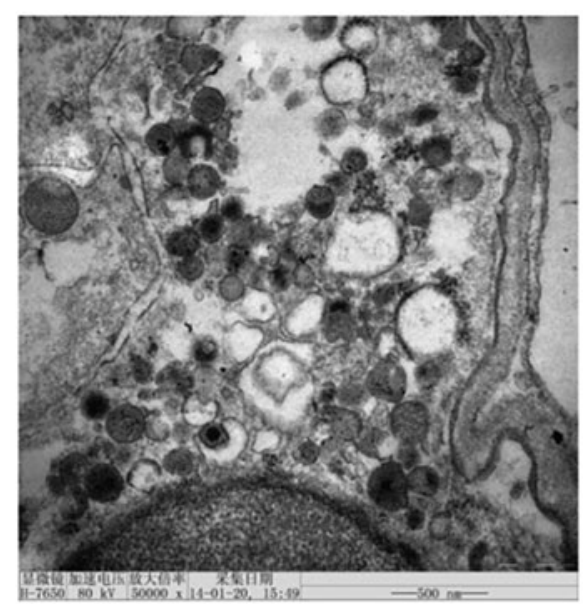

RSV group

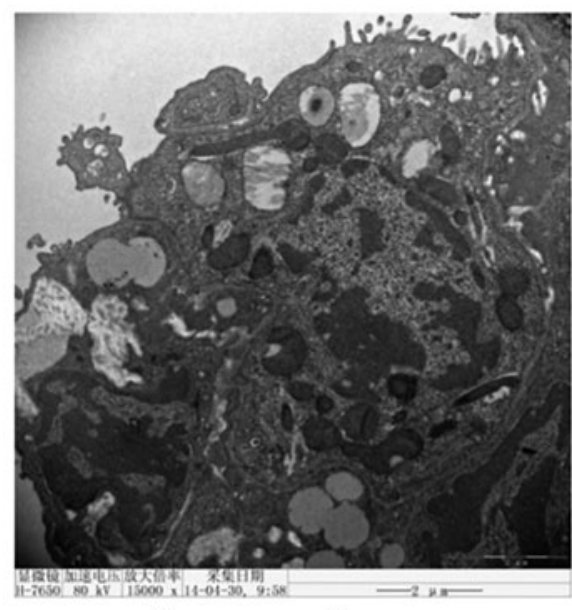

M.vaccae prevention group

FIG. 4. Effects of Mycobacterium vaccae nebulization on RSV infection in the lung of mice. Representative photographs of electron microscope. Lung RSV infection decreased sharply after $M$. vaccae nebulization.

mechanism of asthma. NGF and BDNF are chief members of the neurotrophin family, and play important roles in the regulation of airway neurogenic inflammation. Studies demonstrated that NGF could aggravate inflammation, hyper-responsiveness, and remolding of the airway. AntiNGF treatment may contribute to the prevention and treatment of asthma. ${ }^{(13)}$ BDNF is an important neuroprotection factor produced by pulmonary immune inflammatory cells and airway wall cells. BDNF levels are increased in animal models of asthma as well as in patients with asthma. The structural and functional changes in the airway neural network, that is, the changes in airway neural plasticity, may be connected with the increase in airway hyper-responsiveness. NFs, one of the structural proteins of the airway nerve terminal, are an important element of the neurite skeleton. The quantity and distribution density of NFs indirectly reflect the density of nerve synapses, and thus reflect neural network conditions. Earlier studies have found that transplacental transmission of RSV from mother to offspring can lead to an increase in acetylcholine innervation in fetuses of rats. Further, acetylcholine expression and smooth muscle response to cholinergic stimulation were increased in fetuses of rats reinfected after birth. ${ }^{(14,15)}$

RSV infection does not cause viremia or systemic infection. The viruses merely replicate and proliferate, and stay confined to airway mucosal epithelial cells. Studies have found that EGFR mainly interacts with $2-20 \mathrm{~F}$ protein of RSV, promotes activation of F protein of RSV, and helps in the transmission of RSV infection. ${ }^{(16)}$

TLR7 and TLR8 are intracellular pattern recognition receptors, which can recognize single strand RNA (ssRNA). RSV is a single-strand, negative-strand RNA virus. Thus, both TLR7 and TLR8 play an essential role in RSV infection.

IFN is a low-molecular-weight soluble glycoprotein, divided into types $\alpha, \beta$, and $\gamma$, all of which interfere in virus replication. IFN- $\alpha$ has a notably stronger antiviral effect.

We found that post $M$. vaccae nebulization, the mice in the $M$. vaccae group showed a decrease in pulmonary RSV loading, anti-RSV expression, and RSV mRNA expression level, demonstrating that $M$. vaccae can effectively protect against RSV infection. Further investigation into the mechanism of protection revealed that the 


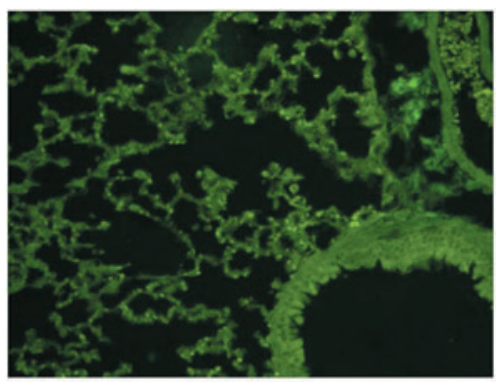

Normal group

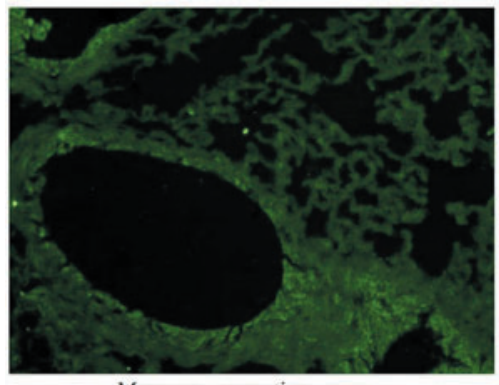

M.vaccae prevention group

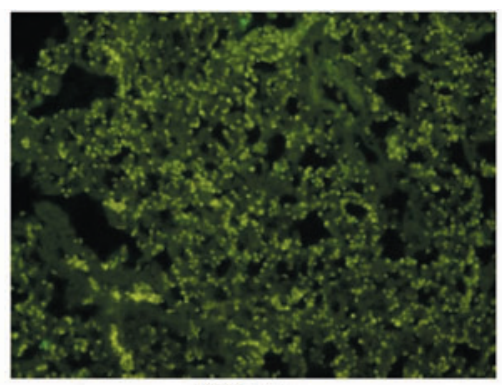

RSV group

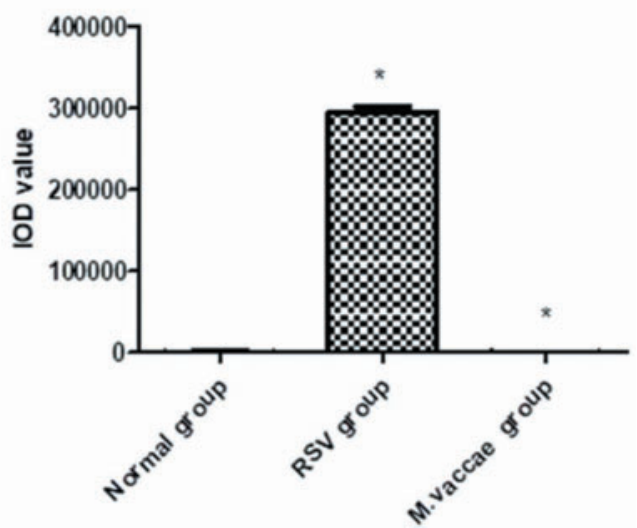

FIG. 5. Effects of Mycobacterium vaccae nebulization on anti-RSV expression in the lung of mice. Representative photomicrographs of anti-RSV immunofluorescence of mice. ( $* p<0.0001$, compared with normal group and RSV group.) Color images are available online.

expression of pulmonary NF09, acetyl choline, and EGFR considerably increased in the RSV group, while it decreased significantly in the $M$. vaccae group. Further, mRNA levels of pulmonary TLR7 and TLR8 increased in the $M$. vaccae group, demonstrating that $M$. vaccae nebulization can protect against RSV infection by regulating neurotransmitters and the expression of TLR7, TLR8, and EGFR.

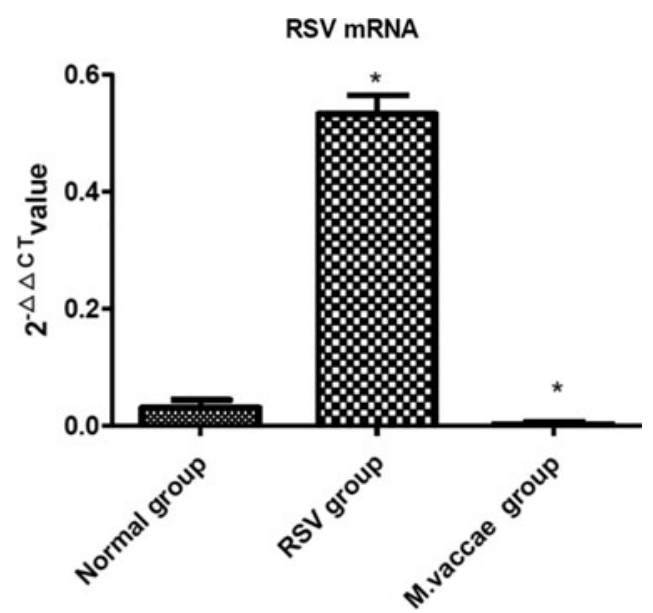

FIG. 6. Effects of Mycobacterium vaccae nebulization on lung RSV mRNA level in mice. $2^{-\triangle \triangle C T}$ value of RSV mRNA detected by fluorescent quantitation polymerase chain reaction. $(* p<0.0001$, compared with normal group; $* * p<0.001$, compared with RSV group.)
Our findings demonstrated that short-time nebulization of M. vaccae could protect against pulmonary RSV infection in Balb/c mice. The mechanism of protection is altogether different from that of a monoclonal antibody vaccine; it involves nonspecific regulation of respiratory antiviral substances-IFNs. In fact, this nebulization seems to be effective against common respiratory viruses such as influenza, parainfluenza, measles, mumps, coronavirus, and avian influenza. Overall, this technique has tremendous potential and is driving further research in our group.

The mechanism underlying airway hyper-responsiveness is known to be complex. ${ }^{(17)}$ Some researchers have shown that the mechanism underlying airway hyper-responsiveness caused by RSV infection is different from that caused by bronchial asthma. ${ }^{(18,19)}$ Our previous studies had confirmed that $M$. vaccae nebulization can reduce the airway hyperresponsiveness and inflammation in the asthmatic model of mice. ${ }^{(7)}$ This study demonstrated that $M$. vaccae nebulization reduced neither the airway hyper-responsiveness nor the airway inflammation caused by the RSV infection. The reason is probably related to the difference between mechanisms of RSV infection and asthma. Therefore, further studies to elucidate this mechanism are required.

To our knowledge, this is the first report on the potential preventive effect of $M$. vaccae nebulization against RSV. Our results showed that $M$. vaccae nebulization has a preventive and treatment effect on RSV infection in mice; however, the underlying mechanism needs to be further studied. The process of nebulization is simple and does not have any side effects, making it easy for patients to carry it 


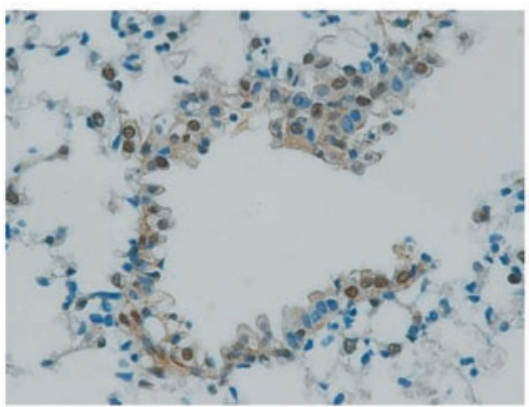

Normal group

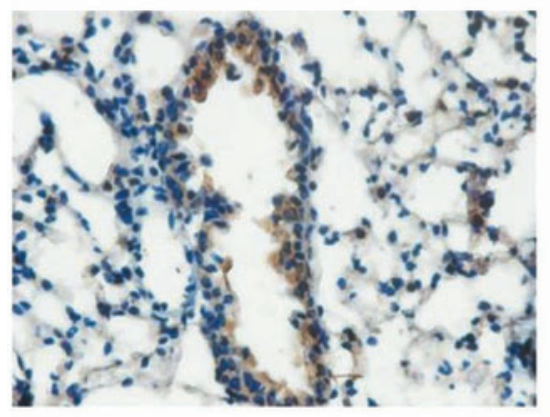

M.vaccae prevention group

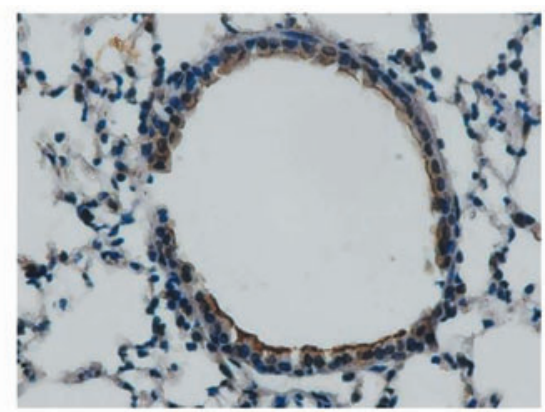

RSV group

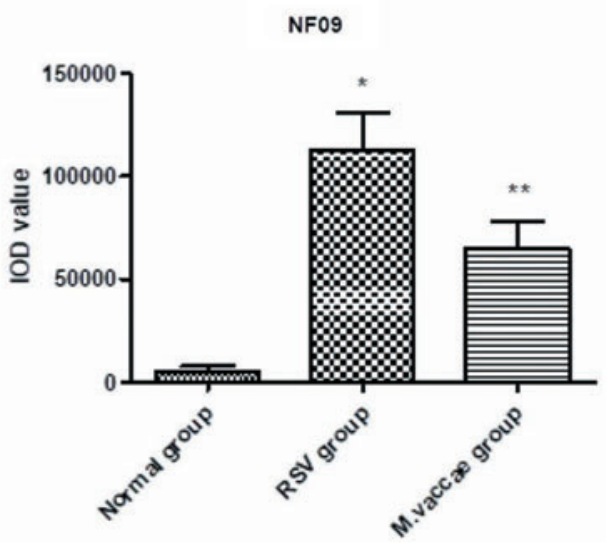

FIG. 7. Effects of Mycobacterium vaccae nebulization on lung NF09 expression in mice. Representative photomicrographs of lung NF09 in mice detected by immunohistochemistry. ${ }^{*} p<0.0001$, compared with normal group; ${ }^{*} p<<0.001$, compared with RSV group. Color images are available online.

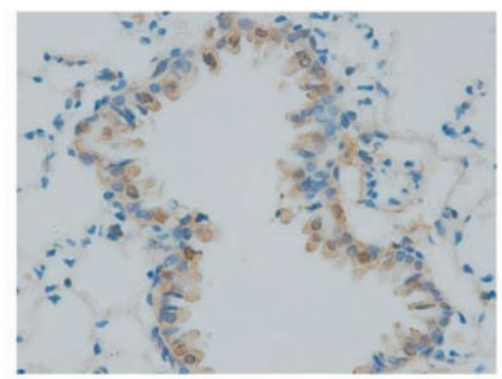

Normal group
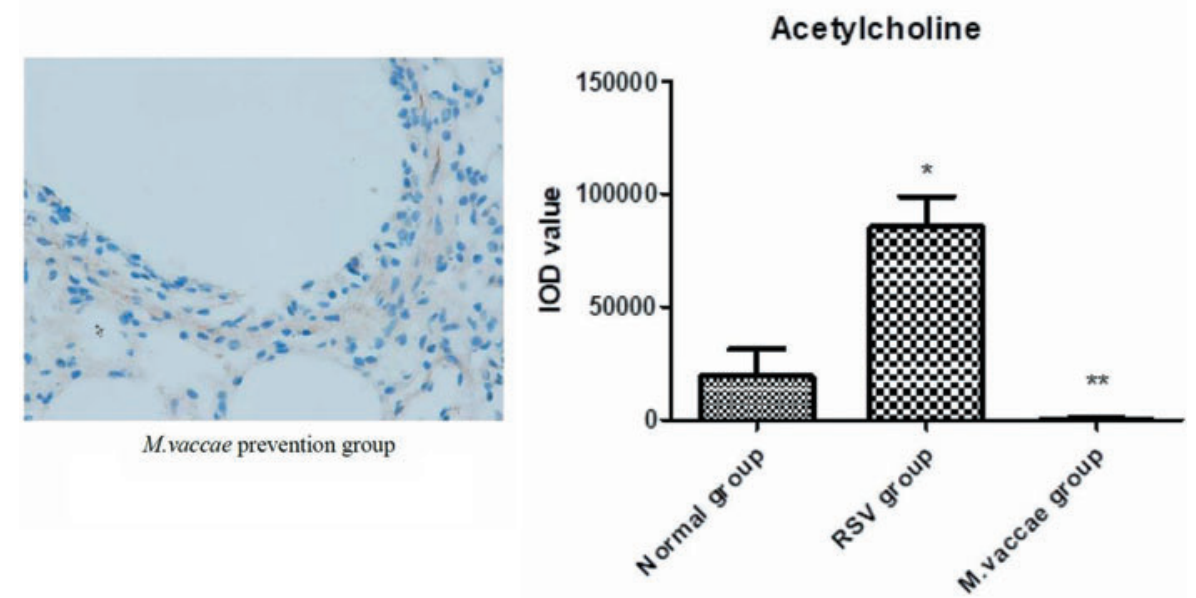

FIG. 8. Effects of Mycobacterium vaccae nebulization on lung acetylcholine expression in mice. Representative photomicrographs of lung NF09 in mice detected by immunohistochemistry. ${ }^{*} p<0.0001$, compared with normal group; $* * p<0.001$, compared with RSV group. Color images are available online. 


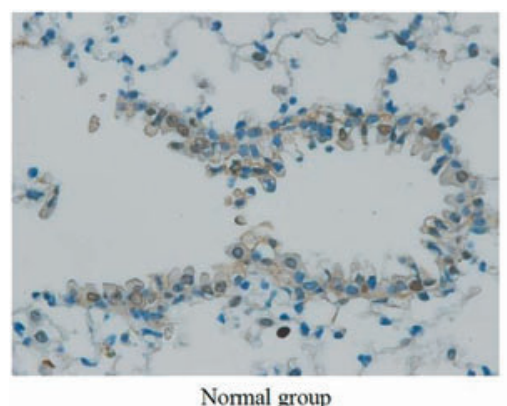

Normal group

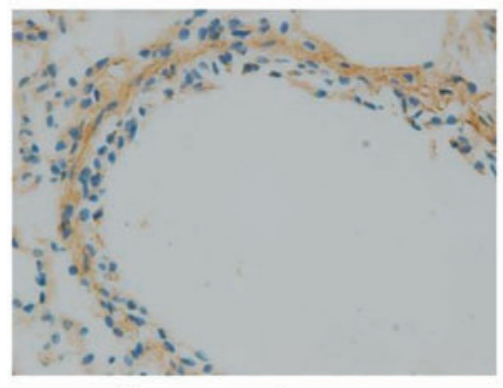

M.vaccae prevention group

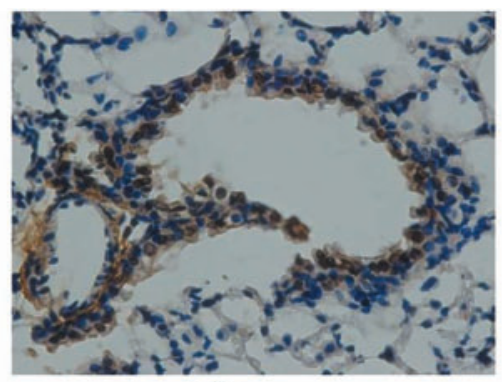

RSV group

EGFR

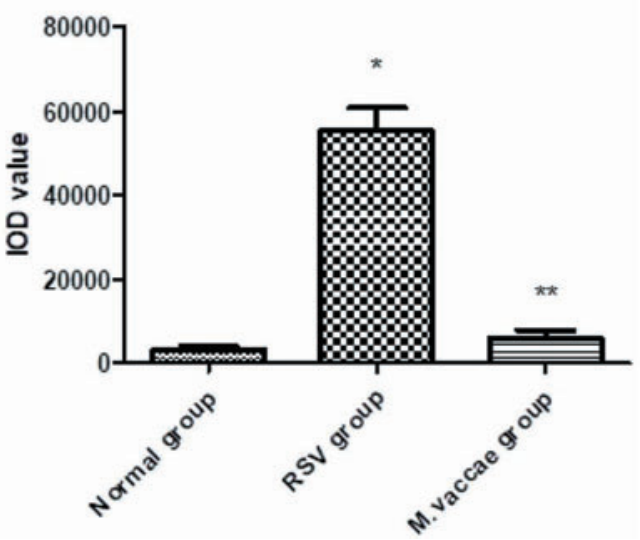

FIG. 9. Effects of Mycobacterium vaccae nebulization on lung EGFR expression in mice. Representative photomicrographs of lung EGFR in mice detected by immunohistochemistry. $* p<0.0001$, compared with normal group; $* * p<0.0001$, compared with RSV group. EGFR, epidermal growth factor regulator. Color images are available online.

out by themselves at their homes. During virus epidemics, such as the 2019 novel coronavirus in Wuhan, China, which is now raging in China, ${ }^{(20)} M$. vaccae nebulization could be used to prevent the spread of the virus. This needs further clinical validation.

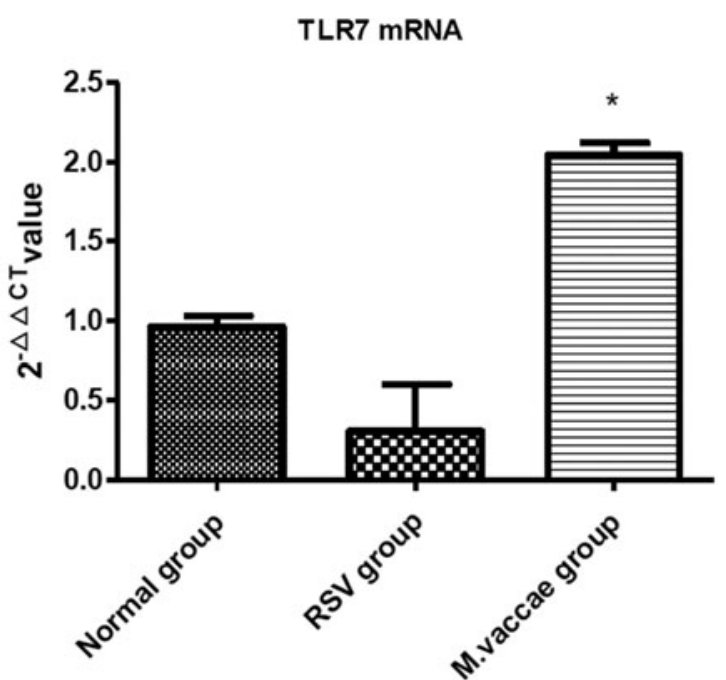

\section{Conclusion}

We showed that $M$. vaccae nebulization could decrease pulmonary RSV mRNA levels in mice. Further research demonstrated that pulmonary NF09, acetylcholine, and EGFR

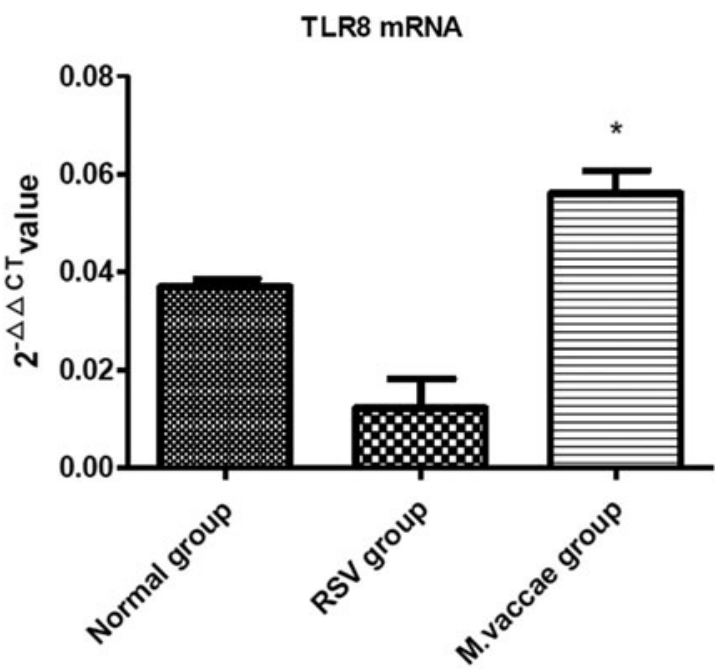

FIG. 10. Effects of Mycobacterium vaccae nebulization on lung TLR7/TLR8 mRNA level in mice. $2^{-\triangle \triangle \mathrm{CT}}$ value of TLR7/TLR8 mRNA level detected by fluorescent quantitation polymerase chain reaction. ${ }^{*} p<0.0001$, compared with RSV group. TLR, toll-like receptor. 
expression decreased considerably, and mRNA levels of TLR7 and TLR8 were increased significantly after $M$. vaccae nebulization.

These results indicate that short-time nebulization of $M$. vaccae can protect against $\mathrm{RSV}$ infection in Balb/c mice. The underlying mechanism involves the regulation of neurotransmitters and expression of TLR7, TLR8, and EGFR. This study will provide new ideas for the prevention of RSV and other viral infections.

\section{Author Disclosure Statement}

The authors declare they have no competing financial interests.

\section{Funding Information}

This work was supported by the following funds: Guangxi Health and Family Planning Commission Self-raised Funds, People's Republic of China (No.: z2014030); Guangxi Medical University Youth Fund, People's Republic of China (No.: XMUYSF201509); and Guangxi University Key Laboratory open issues of emergency medicine, People's Republic of China (No.: GXJZ201601).

\section{References}

1. Jares Baglivo S, and Polack FP: The long road to protect infants against severe RSV lower respiratory tract illness. F1000Res. 2019:8.

2. Simoes EAF, Bont L, Manzoni P, Fauroux B, Paes B, Figueras-Aloy J, Checchia PA, and Carbonell-Estrany X: Past, present and future approaches to the prevention and treatment of respiratory syncytial virus infection in children. Infect Dis Ther. 2018;7:87-120.

3. Gern JE: Mechanisms of virus-induced asthma. J Pediatr. 2003;142:S9-S14.

4. Sigurs N, Gustafsson PM, Bjarnason R, Lundberg F, Schmidt $\mathrm{S}$, Sigurbergsson F, and Kjellman B: Severe respiratory syncytial virus bronchiolitis in infancy and asthma and allergy at age 13. Am J Respi Crit Care Med. 2005;171:137-141.

5. Sigurs N, Aljassim F, Kjellman B, Robinson PD, Sigurbergsson F, Bjarnason R, and Gustafsson PM: Asthma and allergy patterns over 18 years after severe RSV bronchiolitis in the first year of life. Thorax. 2010;65:1045-1052.

6. Kowalewicz-Kulbat M, and Locht C: BCG and protection against inflammatory and auto-immune diseases. Expert Rev Vaccines. 2017;16:1-10.

7. Li C, Jiang X, Luo M, Feng G, Sun Q, and Chen Y: Mycobacterium vaccae nebulization can protect against asthma in Balb/c mice by regulating Th9 expression. PLoS One. 2016;11:e0161164.

8. Prakash YS, and Martin RJ: Brain-derived neurotrophic factor in the airways. Pharmacol Therap. 2014;143:74-86.

9. Tortorolo L, Langer A, Polidori G, Vento G, Stampachiacchere B, Aloe L, and Piedimonte G: Neurotrophin overexpression in lower airways of infants with respiratory syncytial virus infection. Am J Respir Crit Care Med. 2005; 172:233-237.

10. Wegzyn C, Toh LK, Notario G, Biguenet S, Unnebrink K, Park C, Makari D, and Norton M: Safety and effectiveness of palivizumab in children at high risk of serious disease due to respiratory syncytial virus infection: a systematic review. Infect Dis Ther. 2014;3:133-158.
11. Bar-Yoseph R, Haddad J, Hanna M, Kessel I, Kugelman A, Hakim F, and Bentur L: Long term follow-up of Palivizumab administration in children born at 29-32 weeks of gestation. Respir Med. 2019;150:149-153.

12. Ramilo O, Lagos R, Saez-Llorens X, Suzich J, Wang CK, Jensen KM, Harris BS, Losonsky GA, and Griffin MP: Motavizumab treatment of infants hospitalized with respiratory syncytial virus infection does not decrease viral load or severity of illness. Pediatr Infect Dis J. 2014;33:703-709.

13. Yang Y-G, Tian W-M, Zhang H, Li M, and Shang Y-X: Nerve growth factor exacerbates allergic lung inflammation and airway remodeling in a rat model of chronic asthma. Exp Ther Med. 2013;6:1251-1258.

14. Brown PM, Harford TJ, Agrawal V, Yen-Lieberman B, Rezaee F, and Piedimonte G: Prenatal exposure to respiratory syncytial virus alters postnatal immunity and airway smooth muscle contractility during early-life reinfections. PLoS One. 2017;12:e168786.

15. Piedimonte G, Walton C, and Samsell L: Vertical transmission of respiratory syncytial virus modulates pre- and postnatal innervation and reactivity of rat airways. PLoS One. 2013;8:e61309.

16. Lee S, Currier MG, Hotard AL, Meng J, Pretto C, Power UF, Villenave R, Shields MD, Chi MH, Peebles RS, and Moore ML: Epidermal growth factor receptor (EGFR) mediates cell fusion and infectivity of respiratory syncytial virus (RSV). J Allergy Clin Immunol. 2014;133:AB71.

17. Lutchen KR, Pare PD, and Seow CY: Hyperresponsiveness: Relating the intact airway to the whole lung. Physiology. 2017;32:322-331.

18. Song W, Yu Z, Doran SF, Ambalavanan N, Steele C, Garantziotis $S$, and Matalon $S$ : Respiratory syncytial virus infection increases chlorine-induced airway hyperresponsiveness. Am J Physiol Lung Cell Mol Physiol. 2015;309:L205-L210.

19. Zang N, Zhuang J, Deng Y, Yang Z, Ye Z, Xie X, Ren L, Fu Z, Luo Z, Xu F, and Liu E: Pulmonary C fibers modulate MMP-12 production via PAR2 and are involved in the long-term airway inflammation and airway hyperresponsiveness induced by respiratory syncytial virus infection. J Virol. 2015;90:2536-2543.

20. Huang C, Wang Y, Li X, Ren L, Zhao J, Hu Y, Zhang L, Fan G, Xu J, Gu X, Cheng Z, Yu T, Xia J, Wei Y, Wu W, Xie X, Yin W, Li H, Liu M, Xiao Y, Gao H, Guo L, Xie J, Wang G, Jiang R, Gao Z, Jin Q, Wang J, and Cao B: Clinical features of patients infected with 2019 novel coronavirus in Wuhan, China. Lancet. 2020;395:497-506.

Received on September 16, 2019 in final form, March 16, 2020

Reviewed by: Hussein Foda

Address correspondence to: Xiao-Hong Jiang, PhD Department of Geriatric Respiratory Medicine The First Affiliated Hospital of Guangxi Medical University Nanning Guangxi 530021 China

E-mail: 285999574@qq.com 